\title{
Linx
}

Revue des linguistes de l'université Paris X Nanterre

40 | 1999

Le statut d'unité lexicale

\section{Unite lexicale et polylexicalité}

Salah Mejri

\section{OpenEdition}

\section{Journals}

Édition électronique

URL : http://journals.openedition.org/linx/752

DOI : $10.4000 /$ linx.752

ISSN : 2118-9692

\section{Éditeur}

Presses universitaires de Paris Nanterre

\section{Édition imprimée}

Date de publication : 1 juin 1999

Pagination : 79-93

ISSN : 0246-8743

\section{Référence électronique}

Salah Mejri, «Unite lexicale et polylexicalité », Linx [En ligne], 40 | 1999, mis en ligne le 22 juin 2012, consulté le 19 avril 2019. URL : http://journals.openedition.org/linx/752 ; DOI : 10.4000/linx.752

Ce document a été généré automatiquement le 19 avril 2019

Département de Sciences du langage, Université Paris Ouest 


\title{
Unite lexicale et polylexicalité
}

\author{
Salah Mejri
}

1 Les séquences figées (= SF) sont, de par leur morphologie et leur caractère pluriel, des plus problématiques dans ce sens que :

- le statut des SF dans le lexique n'est nullement tranché ; ce qui explique entre autres leur traitement lexicographique souvent très indécis et très peu cohérent et la part qui leur est réservée dans les analyses linguistiques, lexicologiques ou autres, souvent empreintes de flou terminologique et conceptuel, même si l'intérêt qui leur est porté ces dernières années est de plus en plus grand;

- les spécificités des SF en tant qu'unités polylexicales sont loin d'être bien cernées par les différentes théories linguistiques; les descriptions qui en sont faites se limitent le plus souvent aux contraintes syntaxiques qu'elles imposent, à leurs particularités sémantiques, etc.

- le fait qu'elles aient une structure plurielle fondée sur le réemploi d'unités simples conduit le plus souvent à s'interroger sur le statut d'unité lexicale qu'on leur confère intuitivement.

2 Partant de ces considérations, nous voudrions dans ce qui suit nous interroger sur le statut d'unité revendiqué pour les séquences polylexicales et essayer de montrer en quoi résident leurs spécifités linguistiques.

3 Le G.L.L.F. fournit les définitions suivantes du mot unité :

1. Qualité de ce qui forme un tout

2. Qualité de ce qui n'a pas de parties, de ce qui est un (par opposition à pluralité).

3. Caractère de ce qui est continu, suivi, conséquent.

4. Accord, harmonie.

4 Se dégagent de ces définitions les notions de totalité, de continuité, d'harmonie et d'union.

5 Partant de ces définitions, prises dans leur complémentarité, nous pouvons dire que la SF est une unité malgré son caractère pluriel. Cela se justifie indépendamment des caractères linguistiques des SF : seuls les éléments définitoires du mot unité suffisent à conférer à la SF ce statut puisqu'empiriquement (intuitivement), elle est perçue comme 
un tout, continu et dont les constituants sont suffisamment solidaires pour que la pluralité le cède à l'unité.

6 Mais de telles considérations ne sont utiles que pour montrer le caractère approprié du mot unité dans le cas de la SF. En fait, seules les caractéristiques linguistiques nous permettent de trancher dans un sens ou dans l'autre.

7 Dans le cas des $\mathrm{SF}$, on est en présence de séquences pouvant correspondre à n'importe quel type de segment de la chaîne parlée, que ce segment s'intègre dans les différentes parties du discours ou non.

(1) La balle est dans le camp de ...

(2) à la (...)

sont des exemples de séquences qui, tout en étant figées, ne peuvent être versées dans les catégories syntaxiques que sont le nom, l'adjectif, le verbe, l'adverbe, etc.

y ajoutons les unités phrastiques comme les proverbes, la situation se complique davantage parce qu'on sort du cadre de l'unité en tant que « tranche de sonorité qui est, à l'exclusion de ce qui précède et de ce qui suit dans la chaîne parlée, le signifiant d'un certain concept ». (Saussure 1982: 146) ; les proverbes étant des unités jouissant d'une autonomie à la fois syntaxique et sémantique.

9 2. Nous pensons que si nous revenions à la fonction première de la langue qu'est la dénomination, nous pourrions trouver des éléments de réponse à cette contradiction qui fait que des séquences plurielles renfermant des unités simples prétendent malgré cela au statut d'unité. La fonction de dénommer fait que les langues existent, évoluent et s'adaptent aux réalités les plus complexes; elles assurent une telle capacité d'adaptation en puisant :

10 - soit dans les unités non significatives pour créer des signifiants non encore réalisés dans la même langue,

11 - soit dans les unités significatives déjà assignées à des références précises; dans ce dernier cas, deux possibilités sont offertes par le système :

- le réemploi de la même unité simple avec un sens nouveau (le cas de la polysémie ; donc de l'intervention des mécanismes sémantiques),

- le réemploi de plusieurs unités simples versées dans une structure englobante et renvoyant en tant qu'unité complexe à un sens nouveau (Vu sous l'angle de chaque unité simple constituante, ce phénomène ne peut être traité en termes de polysémie ; nous lui préférons le terme de dédoublement (Mejri 1997 b et c, 1998 b et d)).

Les exemples suivants illustrent tous ces cas de figure :

(3) a- halicte, mec.

b- brouhaha, chuchot (er).

(4) a- agiter, dans le sens de "remuer en tous sens".

b- agiter, dans le sens de "troubler moralement..." .

(5) a- casser du sucre sur le dos de quelqu'un, dans le sens littéral.

b- casser du sucre sur le dos de quelqu'un, dans le sens de «dire du mal de lui ». 

l'absence d'éléments significatifs préexistants ayant servi à la formation de l'unité simple, mais ce cas peut englober toutes les unités empruntées ou les unités totalement arbitraires, c'est-à-dire dont le contrat sémiotique entre signifiant et signifié ne porte pas la moindre trace d'éléments médiateurs entre la dénomination et ce qui est dénommé. Aussi paradoxal que cela puisse paraître, les unités d'origine onomatopéique(3b-) relèvent du même principe parce que la médiation sémiotique n'est pas instituée entre le signifiant et le signifié, mais entre le signe linguistique et le référent. Rappelons à ce propos que les onomatopées sont les signes linguistiques où la médiation sémiotique est presque nulle, où le signifiant est en quelque sorte son propre signifié.

Comment peut-on savoir s'il y a médiation ou non dans la construction d'un signe linguistique?

16 3. Si l'on considère que la conceptualisation est une opération fondamentale dans l'appréhension de l'univers, la construction du signe en général, et du signe linguistique en particulier, pourrait en être l'aboutissement ultime; ce qui nous fait dire que l'existence du signe ne peut se faire sans un préconstruit conceptuel. Le concept étant la première opération par laquelle le réel amorphe et fluctuant se trouve transformé en matière pensable, c'est-à-dire transformé en un ensemble de relations structurées entre entités abstraites jouissant d'une très grande généralité servant de repères stables dans la construction de la pensée, il représente une étape nécessaire mais pas suffisante à la construction du signe, lequel peut être linguistique, iconique ou autre ${ }^{1}$.

fois la conceptualisation accomplie intervient le linguistique avec ses deux dimensions signifiante et signifié. Ainsi pourrait-on schématiser ces opérations comme suit :

\begin{tabular}{|l|l|}
\hline Espace idéel & Espace linguistique \\
\hline Univers// conceptualisation & 唒Substance du contenu + éléments \\
\hline & catégoriels + matière phonique \\
\hline (cf. Mejri 1998 c : 43) & \\
\hline
\end{tabular}

18 Ce qui nous autorise à dire que dans ce processus n'intervient pas une médiation particulière du moment que le concept offre à la langue la substance du contenu qui la verse dans ses catégories propres en vue de construire le signifié, lequel s'associe à un signifiant pour donner naissance au signe linguistique.

19 A notre avis, cette analyse n'est valable que pour les unités simples, c'est-à-dire non construites où n'interviennent que le signifé formé à partir de la matière conceptuelle et le signifiant formé directement à partir d'unités phoniques non significatives n'ayant pas servi dans le système linguistique à dénommer d'autres réalités. La raison en est que la conceptualisation se fait dans un espace idéel, c'est-à-dire prélangagier.

Dans le cas des mots construits, dérivés, composés ou figés, la médiation de la langue intervient dans la conceptualisation comme moyen par lequel et à travers lequel le concept se forge. Nous limitons ici notre propos aux SF où, dès le départ, on se sert d'unités linguistiques dotées de leurs propres signifiés et signifiants assurant leur propre fonction dénominative pour renvoyer à d'autres référents. Ainsi la conceptualisation est- 
elle dans ce cas de nature langagière, c'est-à-dire une conceptualisation qui prend son point de départ dans la langue elle-même (Pour les détails de la démonstration, nous renvoyons à Mejri 1998 c : 41-48). Il en résulte un dédoublement qui impose plusieurs autres opérations structurant le sens des SF : globalisation, retrait référentiel, figuration, etc.

Si dans les mots dérivés, les affixes véhiculent des catégories générales, plutôt de nature sémantique ou catégorielle, il n'en est pas de même pour les $\mathrm{SF}$ où les unités constitutives jouissent d'une autonomie sémiotique fondée sur l'immédiateté du contrat entre signifiant et signifié ; mais une fois servant de matière à dénommer une autre réalité, elles connaissent un retrait sémiotique par lequel :

- leur fonctionnement en tant qu'unités autonomes jouissant de leurs fonctions premières (dénomination, référenciation, etc.) se trouve mis en veilleuse au profit de la séquence englobante qui leur sert de cadre dans lequel elles jouent le même rôle que la matière phonique dans le cadre des unités simples. En termes de double articulation du langage, nous dirions que les unités constitutives de la SF se trouvent dans le cadre de la séquence transformées d'unités de la première articulation en unités de la deuxième articulation (cf. Mejri $1998 \mathrm{~d}$ : 3-11), sans qu'il y ait rupture totale entre les deux niveaux d'analyse linguistique ; ce qui autorise entre autres les jeux de mots (cf. Mejri 1997 c : 75-92);

- les opérations sémantiques qui font que la matière langagière déjà engagée dans la conceptualisation de départ serve de substance de contenu au signifié de la SF. Ainsi en est-il de la séquence adjectivale de fer, dans une santé de fer où fer abandonne sa fonction référentielle assurée dans la catégorie du nom au profit de la nouvelle catégorie adjectivale signifiant «la robustesse, la résistance, etc. » et où de verse ce contenu dans une partie du discours non suffixée, instaurant ainsi un nouveau contrat sémiotique entre le signifiant global et le nouveau signifié « robuste, résistant » grâce à la médiation des deux unités de départ de et fer.

Cette globalisation est beaucoup plus perceptible dans les unités polylexicales dont la rupture catégorielle est plus importante comme c'est le cas lors du passage de la catégorie du verbe à celle du nom (séquence hétéro-entité) : dans un couche-tôt, un va-comme -je-tepousse, un va-de-la-gueule, un va-de-l'avant, etc., il est clair que tous les constituants du syntagme verbal de départ, en perdant leur valeur prédicative, se fondent grâce au contenu catégoriel du nom, dans le tout formé par l'unité polylexicale (pour l'opposition entre unités auto- et hétéro-entité, cf. Mejri 1998 : 103-112).

Cette globalisation, qui ramène la pluralité à l'unité, déclenche, grâce au décrochage référentiel des constituants, des opérations abstractives allant de la simple sélection sémique jusqu'à l'extrême subduction qui peut réduire les constituants, de par leur existence phonique, à des éléments dont la simple concaténation produit du sens (cf. Mejri 1994 : 111-122) ; les mécanismes tropiques, tout en étant très fréquents, ne sont pas pour autant nécessaires.

24 Pour résumer, nous dirions que si le statut d'unité lexicale semble évident pour les unités simples (caractère continu, contrat sémiotique immédiat), il semble plus problématique dans le cas des unités complexes, en particulier les SF, et ce en raison de la pluralité des constituants et de la médiation du langage dans la conceptualisation de base. Les opérations évoquées plus haut comme la globalisation² (cf. Mejri, à paraître), l'aréférenciation, la synthèse sémantique (Mejri 1997 a), etc. mises au service de la dénomination d'une nouvelle référence concourent à donner à la SF le statut d'unité. Elles lui assignent ainsi un comportement syntaxique propre conditionnant son insertion dans 
le discours. Ainsi en est-il des différentes contraintes qui opposent les SF aux séquences libres correspondantes :

- contraintes sur la passivation, l'insertion d'items lexicaux entre le verbe et son complément, pronominalisation du complément, etc. pour les séquences verbales comme prendre le large, prendre ses jambes à son cou, prendre la poudre d'escampette, etc. ;

- contraintes sur la nominalisation, sur la coordination, sur la variation paradigmatique, etc. pour les séquences nominales (G. Gross 1997).

Ce qui complique davantage la question, c'est que le figement n'est pas un phénomène absolu ; il se présente, au contraire, sous forme d'un continuum allant du plus figé au plus libre (du plus contraint au moins contraint) nécessitant ainsi une description détaillée du comportement syntaxique des SF ; ce qui est déjà entamé par l'équipe de G. Gross dans le cadre du LLI de Paris XIII (les noms composés, les déterminants, les adjectifs, etc.).

Toujours est-il que ce sont aussi les contraintes qui façonnent l'identité de l'unité. Si on a toujours cru, à tort, que les SF fonctionnent en bloc, c'est parce que leurs comportements syntaxiques qui tranchent en partie avec la syntaxe libre renforcent l'impression de solidarité supposée entre les constituants de la SF. Nous avons essayé de démontrer (Mejri 1997 a) que le comportement syntaxique n'est que la conséquence logique de la structuration sémantique de la séquence et que l'intervention des mécanismes tropiques renforce les contraintes syntaxiques (Mejri 1998 e : 103-112).

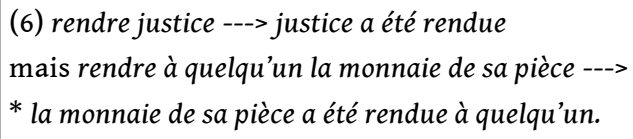

Ce qui signifie que plus la rupture avec les traits sémantiques et syntaxiques des unités de base est importante, plus l'unité de la SF est perceptible.

4. Faisons une dernière remarque relative aux séquences dites familières avant d'entamer les proverbes : il nous a été donné de constater lors de l'étude de l'arabe tunisien que le nombre des SF est aussi important que les séquences libres alors qu'il n'en est pas de même pour l'arabe dit littéral ou classique où seules quelques séquences persistent dans son emploi actuel alors que les anciennes descriptions lexicographiques montrent qu'il était aussi riche que les langues actuellement en usage régulier par les communautés linguistiques. Cette remarque est importante parce qu'elle permet de mieux apprécier la fonction expressive du langage : plus la langue est vernaculaire, plus elle est expressive, plus le recours aux SF est important.

Le propre de ces séquences, c'est qu'elles forment le plus souvent avec les unités lexicales équivalentes de la langue standard une sorte de «doublets » qui renvoient aux mêmes concepts. Notre objet n'est pas de voir leur structuration sémiotique (cf. G. Petit 1998 : 145-163) mais de montrer comment le même référent peut faire l'objet de deux conceptualisations différentes: l'une est conçue dans un espace idéel, l'autre dans un espace langagier.

On a souvent considéré comme des synonymes des unités lexicales n'appartenant pas au même registre, parce qu'elles renvoient au même référent, or le renvoi à la référence ne s'y fait pas par les mêmes voies. Si nous empruntons au Dictionnaire de l'argot les équivalents locutionnels de haschisch, c'est-à-dire fée verte et piano du pauvre, nous verrons que les trois dénominations n'ont pas été construites selon le même principe : 
31 - dans hasahisch, d'origine arabe (= "herbe »), le contrat sémiotique est immédiat puisqu'on est passé de la construction du concept à la formation du signe linguistique (signifiant et signifié) ; le résultat étant le rapport signifiant [a] if ] / signifié « drogue... ».

32 - dans fée verte ou piano du pauvre, la conceptualisation se fait au moyen des différents items des deux séquences avec leurs charges à la fois sémiotiques, sémantiques, référentielles, paradigmatiques, etc. Cette intervention du langage à une étape normalement prélangagière fait que les items qui servent de médiateurs entre la conceptualisation et la formation du signe linguistique conditionnent le signe linguistique d'aboutissement du moment que le linguistique préexiste à cette nouvelle formation lexicale.

33 En d'autres termes, dans le premier cas, puisque le linguistique n'est que l'aboutissement de toute l'opération, le choix du signifiant est nécessairement arbitraire (ou onomatopéique); dans le second, puisqu'il intervient dès la conceptualisation, il continue à agir sur la suite de l'opération; ce qui donne lieu à une séquence dont la teneur sémantique est loin d'être quelconque puisque la SF est fondée sur un dédoublement dont les niveaux pourraient être décrits à partir des exemples cités comme suit :

- niveau des unités simples prises séparément : fée, verte, piano, du et pauvre avec tout ce que cela comporte comme rapports paradigmatiques divers et comme valeurs diverses, connotations ou autres ;

- niveau des syntagmes libres avec leurs sémantismes propres, leur syntaxe et leurs particularités discursives ;

- niveau de la SF qui, moyennant une synthèse sémantique, assure la fonction dénominative en mettant en veilleuse les autres niveaux.

C'est cette épaisseur qui assure la fonction expressive mentionnée plus haut et qui fait que ceux qui maitrisent le niveau standard éprouvent le besoin d'employer d'autres moyens langagiers jugés plus adéquats.

Ainsi pourrions-nous schématiser les deux processus de la manière suivante :

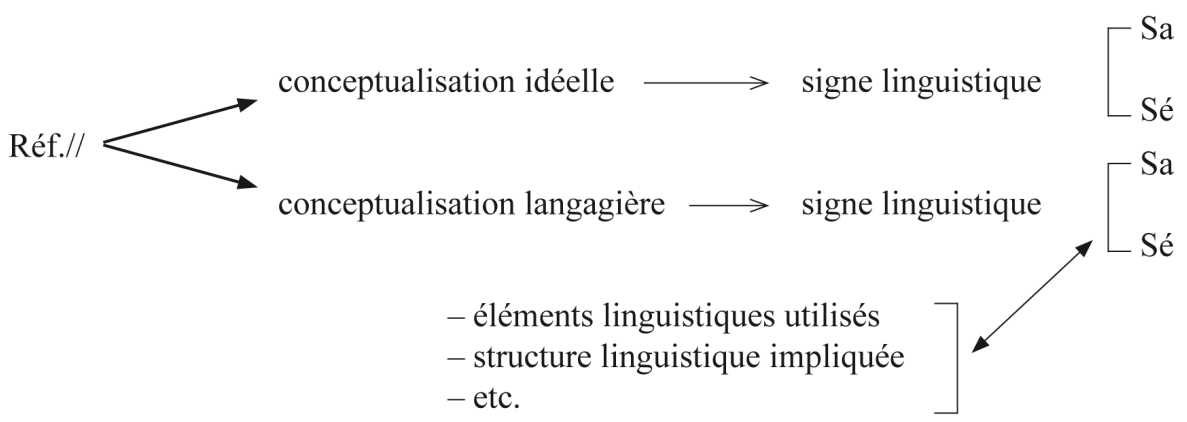

5. S'agissant des proverbes maximes, sentences, adages, phrases génériques, etc.), il y a lieu de constater que ce type de SF partage avec les autres toutes les caractéristiques décrites plus haut, à l'exception de l'appartenance à une partie du discours qui détermine en grande partie le comportement syntaxique de la séquence. Avec le proverbe, il s'agit de séquences dont la syntaxe ne dépend pas d'un élément intégrant et dont le sens ne cherche pas sa complétude dans un cadre plus englobant. En toute logique, il ne peut pas faire partie du lexique ; les SF ont une taille qui correspond généralement aux syntagmes, celle des proverbes pose le problème de la phrase : est-elle une unité de langue ou une unité de discours? Si on optait pour le discours, les proverbes ne feraient pas partie de notre propos. Si au contraire on considérait les proverbes comme des faits de langue, il 
faudrait trouver des réponses à des questions se rapportant au statut de préconstruit que présuppose l'appartenance à la langue, et dans le cas où on lui accorderait le statut d'élément de langue, que serait l'englobant dans lequel il s'insère pour que le principe de l'intégration des éléments hiérarchiquement inférieurs dans des unités supérieures soit respecté. Aucun autre choix que le texte ne semble possible dans ce cas. sens qu'il est un répertoire qui sert à rendre compte du monde, il n'y a aucun doute que les proverbes, malgré leur statut de phrase ${ }^{3}$, dénomment des concepts, même si ces concepts sont d'un type particulier, c'est-à-dire des attitudes, des comportements, des façons d'être, etc. (cf. Kleiber 1998). Dire :

(7) La nuit, tous les chats sont gris,

La plus belle fille du monde ne peut donner que ce qu'elle a,

c'est exprimer « la difficulté de discernement » et « les limites objectives dans lesquelles on se trouve ». Partant de ce constat, les dictionnaires commencent à rompre avec l'attitude de rejet qu'ils ont souvent observée à l'égard des proverbes: le G.L.L.F. par exemple cite dans l'article chat trois proverbes dont le statut est explicitement mentionné : A bon chat, bon rat; Quand le chat n'est pas là, les souris dansent; Chat échaudé craint l'eau froide.

Quant aux relations d'équivalence avec les unités du lexique, elles sont déjà impliquées dans la remarque précédente puisque les proverbes font l'objet de descriptions lexicographiques les mettant en rapport avec des paraphrases ou des équivalents lexicaux: parler de méfiance ou dire chat échaudé craint l'eau froide, c'est renvoyer globalement au même concept mais avec cette différence que l'énoncé proverbial possède une prégnance plus grande provenant de la nature oblique de la dénomination qu'il assure : c'est-à-dire une dénomination qui, au lieu de puiser dans le lexique les termes 
servant à désigner directement les entités dénommées, se sert d'unités lexicales renvoyant à d'autres réalités.

En ce qui concerne la structuration sémantique, malgré l'idée couramment admise selon laquelle les proverbes énoncent des vérités d'expérience, on peut dire que les proverbes, tout comme les unités du lexique, entretiennent des relations :

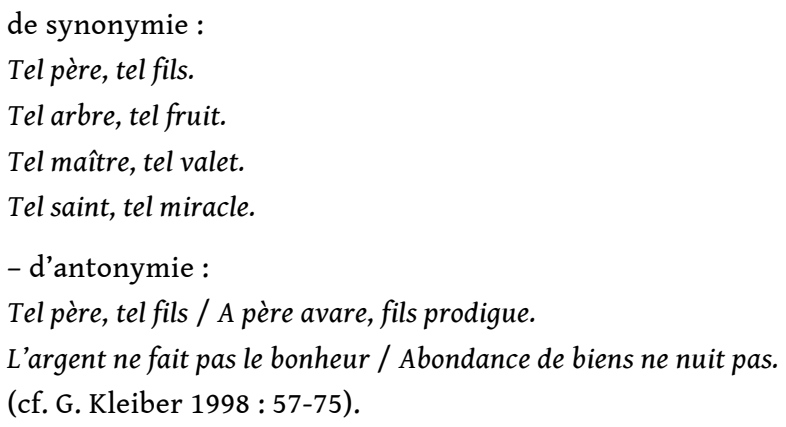

Une dernière remarque: si les SF de dimension infra-phrastique traduisent leurs contraintes syntaxiques dans le cadre de la phrase, le proverbe se réalise dans un espace plus important, l'espace textuel où il joue le rôle de catalyseur sémantique et où il assure la structuration de l'énoncé; d'où sa valeur emblématique et la fréquence de ses emplois dans les titres (cf. Mejri 1997 a).

6. Partant de toutes ces considérations, nous pouvons avancer les éléments de réflexion suivants : a- La dénomination, qui est la fonction première des unités lexicales, est assurée par tous les types de SF, quelles qu'en soit la dimension et la nature.

b- Les SF s'opposent aux unités simples par leur caractère pluriel, et par conséquent par l'intervention du linguistique dans l'opération de conceptualisation à l'origine de la formation du signe linguistique polylexical ; ce qui leur donne une saillance linguistique et une prégnance sémiotique souvent perçues intuitivement comme «charge connotative ", " saveur idiomatique », ou " valeur stylistique ».

c- Le dédoublement propre aux SF fait que ce type d'unités lexicales, tout en fonctionnant au niveau du système comme des unités globales, gardent toujours dans le discours la possibilité de réactivation des charges sémantiques et du fonctionnement des constituants parce que le signifiant global de la SF est le foyer d'une synthèse sémiotique où le constituant, de par l'oblicité dénominative, désigne autre chose que ce qu'il dit tout en conservant toutes ses potentialités premières. Dans un ange passe, on parle bel et bien d'un ange et du prédicat verbal passer mais on signifie « un silence prolongé dans une assemblée ».

d- C'est cette rupture sémiotique constatée au niveau des constituants qui fonde l'unité globale et lui donne son vrai statut d'unité.

\section{Pour conclure :}

49 Nous ne croyons pas avoir évoqué tous les mécanismes régissant les SF ; telle n'est pas notre intention; nous avons essayé de montrer que, contrairement à une tradition bien 
ancrée dans les approches linguistiques, les unités polylexicales sont réellement des unités du lexique malgré leur caractère pluriel, et qu'elles doivent ce statut à la nature des opérations complexes que leur création implique (conceptualisation langagière, oblicité dénominative, globalisation, rupture sémiotique, aréférenciation, synthèse sémantique, figuration, etc.) et qui conditionnent leur fonctionnement syntaxique dans le cadre de la phrase (pour les unités infraphrastiques) et dans un cadre textuel (pour les unités phrastiques comme les proverbes).

Encore faut-il préciser que ces analyses s'appliquent aussi bien au français qu'à l'anglais ou l'arabe (cf. M. Baccouche Ghariani 1998 : 329-342).

Il reste à démontrer :

- si l'oblicité dénominative et la conceptualisation langagière sont systématiquement en corrélation. Si tel est le cas, le mécanisme tropique sera l'axe autour duquel s'articulera l'étude des SF. Nous penchons du côté de l'opinion inverse dont nous avons esquissé les contours à propos des proverbes (Mejri 1997 a) mais dont l'analyse plus détaillée appliquée à un grand corpus aiderait à montrer comment cette non-systématicité de la corrélation fonde le contrat sémiotique établi entre un signifiant pluriel et un signifié global ;

- si le statut d'unité fondé sur les opérations déjà évoquées peut être cerné au moyen de critères assez opérationnels pour permettre une reconnaissance automatique dans le discours; nous pensons qu'un traitement de corpus conçu sur la base de la notion de dédoublement qui présuppose que toute séquence est susceptible d'au moins un emploi autre que son emploi régulier pourrait fournir le moyen de concevoir une approche intégrant le principe de l'analyse en paliers selon laquelle toute séquence serait analysée en tant que séquence compositionnelle (l'unité est d'ordre plutôt lexémique ou «morphémique »(4)) puis syntagmatique (compositionnelle ou séquencielle $(5)$ ), puis phrastique (compositionnelle ou dénominative); le tout étant conçu sur la base de la lexicalisation de ces unités polylexicales ;

- si le dédoublement n'est pas à l'origine de la structuration autonymique des SF qui aboutit à la formation des outils syntaxiques (grammaticaux) dont peut disposer une langue.

Beau programme pour les linguistes !

\section{BIBLIOGRAPHIE}

BACCOUCHE T. et MEJRI S., Du mot, Dar El Janoub, Tunisie, 151 p. (en arabe).

BACCOUCHE T., MEJRI S. et BACCOUCHE GHARIANI M., (1998) : « Du sacré au profane : le parcours d'une lexicalisation ", in Le figement lexical, sous la dir. de S. Mejri, G. Gross, A. Clas et T. Baccouche, Tunis 1998, pp. 121-131.

BACCOUCHE GHARIANI M. (1998) : « Formules de salutation, d'une langue à l'autre. Classement et enseignement », in Le figement lexical, sous la dir. de S. Mejri et ali, Tunis, 1998, pp. 329-342.

COLIN J-P., MEVEL J-P et LECLRE Chr., Dictionnaire de l'argot, Larousse, 1990. 
GRECIANO G. Signification et dénotation en allemand. La sémantique des expressions idiomatiques. Recherches linguistiques, Etudes publiées par le Centre d'analyse syntaxique, Université de Metz, IX, Faculté des lettres et sciences humaines, 1983, 469 p.

GROSS G. (1996) : Les expressions figées en français, Orphys, Paris 1996.

KLEIBER (G.) : « Les proverbes antinomiques : une grosse pierre « logique », dans le jardin toujours « universel des proverbes ", in Le figement lexical, sous la direction de S. Mejri, G. Gross, A. Clas et T. Baccouche, $1^{\text {ère }}$ R.L.M., CERES, Tunisie 1998, pp. 51-75.

MEJRI (S.) (1994) : « Séquences figées et expression de l'intensité. Essai de description sémantique », Cahiers de lexicologie, 65, 1994-2/, pp. 111-122.

(1995) : La néologie lexicale. Publications de la Faculté des lettres de la Manouba, Tunisie, 1995, 380 p.

(1997 a) : Le figement lexical. Descriptions linguistiques et structuration sémantique. Publications de la Faculté des lettres. La Manouba, Tunisie, 1997, 632 p.

(1997 b) : «Binarisme, dualité et séquences figées », in Les formes du sens, Mélanges Robert Martin, Duculot, 1997, pp. 249-256.

(1997 c) : « Défigement et jeux de mots », Etudes linguistiques, vol. 3, Association Tunisienne de Linguistique, Tunis 1997, pp. 75-92.

(1998 a) : « Du figement lexical : continuité référentielle et saillance linguistique », Scolia 11, Strasbourg, 1998, pp. 169-179.

(1998 b) : « Le figement et la linéarité du signe linguistique », L'information grammaticale, numéro spécial « Tunisie », mai 98, Paris, pp. 17-21.

(1998 c) : « La conceptualisation dans les « séquences figées », id. pp. 41-48.

(1998 d) : «La mémoire des séquences figées : une troisième articulation ou la réhabilitation du culturel dans le linguistique », Actes du colloque La mémoire des mots, sous la direction de A. Clas, S. Mejri et T. Baccouche, Actualité scientifique, AUPELF-UREF, Tunis, 1998, pp. 3-11.

(1998 e) : «Structuration sémantique et variation des séquences figées », in Le figement lexical, 1 ère Rencontre Linguistique Méditerrannéenne, Tunis les 17-18 et 19 sept. 98, sous la direction de $S$. Mejri, G. Gross, A. Clas et T. Baccouche, Tunis 1998, pp. 103-112.

(1998f) : Le figement lexical, $1^{\text {ère }}$ Rencontre Linguistique méditerrannéenne, Tunis, 1998, 362 p. PETIT G. « Remarques sur la structuration sémiotique des locutions familières» in Le figement lexical, sous la dir. de S. Mejri, G. Gross, A. Clas et T. Baccouche, Tunis 1998, pp. 145-163.

PRANDI M. (1992) : Grammaire philosophique des stropes, Minuit, Paris, 1992.

(1998) : «Les motivations conceptuelles du figement », in Le figement lexical, sous la dir. de S. Mejri et ali-, Tunis, 1998, pp. 87-101.

SAUSSURE F. de Cours de linguistique générale, Payot, 1982.

\section{NOTES}

1. Il arrive même que le même concept soit traduit dans deux ou plusieurs codes comme c'est le cas en géométrie où on peut avoir la figure, sa traduction formelle et sa description en langue naturelle. 
2. La globalisation est définie par G. Gréciano comme étant « un processus de réunion à la fois sélective et virtuellement illimitée des composantes propres aux parties auparavant disparates en une unité » (1983: 387).

3. Faut-il remarquer que ce caractère ambivalent n'est pas propre au proverbe : les autres SF sont à la fois une dénomination et une séquence libre (syntagme verbal, nominal, prépositionnel, etc.). 4. Pour la clarté de l'exposé, nous avons évité intentionnellement la problématique du mot à laquelle nous avons consacré une étude (cf. bibliographie).

5. C'est-à-dire relative aux SF.

\section{RÉSUMÉS}

Les séquences figées, qui sont par définition polylexicales, ne sont pas considérées comme des unités en raison de cette polylexicalité même. Or leur fonction dénominative, leur structuration sémantique et leur intégration dans le lexique en font des unités lexicales ayant un fonctionnement global qui en indique les spécificités et en façonnent l'identité. Les concepts de conceptualisation, de globalisation et de dénomination obliques aident à montrer comment des séquences figées aussi différentes que les syntagmes et les proverbes peuvent prétendre au statut d'unité.

Fixed phrases, wich are by definition multiword units or polylexical units, are not considered as units because of this very polylexicality. But their denominative function, their semantic structuring and their integration into the lexis make of them lexical units having a global functioning which indicates their specifities and shapes their identity. The concept of conceptualization, globalization and oblique denomination help to show how fixed phrases as different phrase and proverbs can lay claim to the status of unit.

\section{AUTEUR}

\section{SALAH MEJRI}

Institut Supérieur de l'Education et de la Formation Continue

43, rue de la liberté -Le Bardo 2019 Tunis

Tunisie 